\title{
The Journey of a Prescription: Authentic Interprofessional Education Simulation of the Patient and Prescription Flow through Prescriber and Dispenser
}

\author{
James A. Green ${ }^{1,2^{*}}$, Jim Ross ${ }^{1}$, Rhiannon Braund ${ }^{1}$, Martyn Williamson ${ }^{1}$, Tony \\ Egan $^{1}$ \\ 1 University of Otago, Dunedin, New Zealand \\ ${ }^{2}$ School of Allied Health and Physical Activity for Health Research Cluster, Health \\ Research Institute (HRI), University of Limerick, Ireland
}

There is an increasing emphasis on interprofessional education for health professional students. However, exercises at the immersion level - where students are embedded in their professional roles - are challenging to design. We investigated the feasibility of running a series of simulated pharmacies alongside an existing simulated medical clinic. Students enjoyed the added authenticity of interprofessional simulation, but that this made them feel they had to represent their profession well. Simulation suites for different health professions should be co-located where possible.

\section{Background}

Simulation is commonly used in both Medicine and Pharmacy teaching to enable experiential learning in a safe and controlled way, usually relating to individual components of the clinical role (e.g. in Pharmacy, dispensing or patient counselling). Dunedin General Practice teaching staff have integrated the component-based use of

\footnotetext{
* Corresponding author: Dr James Green, School of Allied Health, Health Sciences Building, University of Limerick, Ireland V94 T9PX, lames.Green@ul.ie, +35361234987
}

Authors and contributions: JG and JR came up with the original idea, and developed the IPE session along with RB, MW and TE. JG wrote the initial draft, which was revised following feedback from JR, RB, MW and TE. All authors approved the final draft and agree to be accountable for all aspects of this work.

Funding: This project received funding from the University of Otago Interprofessional Education Support Innovation Fund (112016IPEF).

Conflict of Interest: None

Statement of Ethical Approval: This project was approved by the University of Otago Human Ethics Committee (17-007).

Data Availability: We have consent to share anonymised transcripts of the focus groups/debrief with other researchers on request. 
simulation to develop a teaching method known as the SECO Clinic ('Safe and Effective Clinical Outcomes' for patients) (Williamson et al., 2013). The students adopt the role of doctor and conduct entire consultations. After each 2-hour clinic students mark their performance against pre-specified outcomes for each case and receive written feedback from simulated patients, relevant evidence -based information and a group debrief. End-of-attachment reflective essays indicate that the SECO Clinic is a powerful learning environment for students, focusing previous clinical skills learning and teaching clinical problem solving experientially (Young, Williamson, \& Egan, 2016).

\section{Intended learning outcomes}

1. Through this interprofessional activity medical and pharmacy students would practise their roles in the safe and effective use of medication, including expanding their understanding of each profession's roles and issues.

2. Medical students would integrate prescription writing and communication with pharmacists into their delivery of safe and effective care for simulated patients.

3. Pharmacy students would communicate with simulated patients and prescribers and dispense medication in order to achieve safe and effective medication use.

\section{What was tried?}

8 final (fourth) year pharmacy students operated 4 simulated pharmacies serving a simulated medical practice with 18 fourth year medical students working in pairs, and 23 actors serving as patients. The simulated medical centre is purpose built and includes a waiting room, reception, and ten offices. Pharmacies were set up in nearby tutorial rooms, with pharmacy computer systems, label printers, and sufficient stock for anticipated prescriptions. Medical and pharmacy students communicated via landline or mobile telephones as required, routed through the medical centre reception.

Simulated patients (SPs) were initially seen at the medical centre, then wrote formative feedback for the medical students. Each SP then took the prescription to a pharmacy to receive their medication. The SP then wrote formative feedback for the pharmacy students, and also compared the information and advice given by the medical and pharmacy students. Following 2 hours of simulation, students participated in a focus group/debrief in mixed profession groups.

\section{What lessons were learned?}

Overall, the session ran smoothly, despite the considerable complexity in setting up temporary pharmacies with computer software and stock, and in managing the flow 
of patients and prescriptions between the medical and pharmacy settings. We hosted observers from physiotherapy and medical laboratory science, and are exploring how these professions could be integrated in future simulations.

We had hoped, based on our prior research (Green, Ross, \& Stubbe, 2016; Trausch \& Green, 2018), that the students would be able to readily engage each other by telephone, as they do in real practice. However, only a couple of these calls occurred. The logistics of this was not as well realised as we had hoped (leading to a last minute switch to mobile phones). Some preparation for these calls in advance might also have lowered barriers to initiating these interactions.

Because of the need for actors to move from simulated medical centre to simulated pharmacy, the existing simulated pharmacy was too far from the simulated medical centre. This necessitated the setting up of more temporary pharmacy spaces, with limited stock and resources. Co-locating simulation suites for different health professions would facilitate future interprofessional education initiatives.

Analysis of focus group/debrief transcripts showed that students found the experience beneficial and more authentic than their usual simulation experiences. They comment on learning a lot about each other's roles, and the opportunity to spend extended time in role themselves:

"It was more like real life" [medical student]

"[in pharmacy] we'll do the dispensing aspect, or we'll do the communication aspect; we don't often do the whole process in one hit, let alone do that whole process and have real prescribers sending [prescriptions] down." [pharmacy student]

"So, it's good to have this kind of exercise, and we will work better in the future if we understand what each other does. So, in the future if there was an option there for us to talk to them pharmacists and build our prescription and get some immediate - direct feedback I should say, about for a particular case, and maybe the patient had questions we didn't answer, or maybe we didn't do the right thing on the prescription" [medical student]

However, both groups of students were aware of the potential for judgement from the other group: 
"I found it quite daunting writing a prescription that was going to a

pharmacist" [medical student]

This pilot provides a model for further interprofessional education development. Through the simulation exercise and debrief discussion, both groups of students were able to learn about each other's roles in obtaining good outcomes for their shared patients.

\section{References}

Green, J. A., Ross, J., \& Stubbe, M. (2016). Spoken and written interaction between community pharmacy staff and general practitioners [family physicians]. International Journal of Pharmacy Practice, 24, 14.

Trausch, N., \& Green, J. A. (2018). Direct observation of telephone communication between community pharmacies and prescribers in New Zealand. International Journal of Clinical Pharmacy, 1-5. doi:10.1007/s11096-018-0687-8

Williamson, M., Walker, T., Egan, T., Storr, E., Ross, J., \& Kenrick, K. (2013). The safe and effective clinical outcomes (SECO) clinic: learning responsibility for patient care through simulation. Teaching and learning in medicine, 25(2), 155-158. doi:10.1080/10401334.2013.772016

Young, J. E., Williamson, M. I., \& Egan, T. G. (2016). Students' reflections on the relationships between safe learning environments, learning challenge and positive experiences of learning in a simulated GP clinic. Advances in Health Sciences Education, 21(1), 63-77. doi:10.1007/s10459-015-9611-3 